\title{
Lateral roots affect the proteome of the primary root of maize (Zea mays L.)
}

\author{
Frank Hochholdinger ${ }^{1,5, *}$, Ling Guo ${ }^{2,3}$ and Patrick S. Schnable e $^{1,2,3,4}$ \\ ${ }^{1}$ Department of Agronomy, Iowa State University, Ames, IA 50011-3650, USA (*author for correspondence; \\ e-mail frank.hochholdinger@zmbp.uni-tuebingen.de); ${ }^{2}$ Department of Genetics, Development, and Cell \\ Biology, Iowa State University, Ames, IA 50011-3650, USA; ${ }^{3}$ Bioinformatics and Computational Biology \\ Graduate Program, Iowa State University, Ames, IA 50011-36506, USA ${ }^{4}$ Center for Plant Genomics, Iowa \\ State University, Ames, IA 50011-3650, USA; ${ }^{5}$ Present address: Center for Plant Molecular Biology \\ (ZMBP), University of Tuebingen, Auf der Morgenstelle 28, 72076 Tuebingen, Germany
}

Received 17 August 2004; accepted in revised form 20 September 2004

Key words: lrt1, maize, mutant, proteome interactions, proteomics, roots

\begin{abstract}
Lateral roots are initiated from the pericycle cells of other types of roots and remain in contact with these roots throughout their life span. Although this physical contact has the potential to permit the exchange of signals, little is known about the flow of information from the lateral roots to the primary root. To begin to study these interactions the proteome of the primary root system of the maize (Zea mays L.) lrtl mutant, which does not initiate lateral roots, was compared with the corresponding proteome of wild-type seedlings 9 days after germination. Approximately 150 soluble root proteins were resolved by two-dimensional electrophoresis and analyzed by MALDI-ToF mass spectrometry and database searching. The 96 most abundant proteins from a $\mathrm{pH} 4-7$ gradient were analyzed; 67 proteins representing 47 different Genbank accessions were identified. Interestingly, $10 \%(15 / 150)$ of the detected proteins were preferentially expressed in $l r t 1$ roots that lack lateral roots. Eight of these $l r t 1$-specific proteins were identified and four are involved in lignin metabolism. This study demonstrates for the first time the influence of lateral roots on the proteome of the primary root system. To our knowledge this is the first study to demonstrate an interaction between two plant organs (viz., lateral and primary roots) at the level of the proteome.
\end{abstract}

\section{Introduction}

The root system of maize (Zea mays L.) comprises embryonically formed primary and seminal roots, as well as post-embryonic shoot-borne crown and brace roots (Hochholdinger et al., 2004a). A common characteristic of all root types is the formation of lateral roots. Lateral roots play an important role in root stock architecture (Lynch, 1995) and are responsible for the majority of water and nutrient uptake by maize plants (McCully and Canny, 1988; Varney and Canny, 1993; Wang et al., 1991, 1994, 1995). Lateral roots are initiated in the differentiation zone of the main root some distance away from the root apex where pericycle cells are no longer actively dividing (Esau, 1965). Developing main roots constantly proliferate new lateral roots. Thus, the longitudinal positions of lateral roots along the main root indicate their developmental status, with the younger lateral roots located closer to the root tip. The root tip region contains also many newly initiated lateral root primordia that have been initiated but are not yet visible.

Few mutants that affect lateral root formation have been isolated in the monocotyledonous cereals (Hochholdinger et al., 2004a) with only one mutant (lrt1) affected in the initiation of lateral roots. The $\operatorname{lr} t 1$ mutant is specifically affected 
in the early post-embryonic phase in failing to initiate lateral roots from the embryonic primary and seminal roots and crown roots from the coleoptilar node (Hochholdinger and Feix, 1998).

Each individual main root and its lateral roots represents a small 'root module' consisting of hundreds of lateral roots that must interact closely with the main root to which they are connected. Signaling between main roots and lateral roots plays a pivotal role for the formation of the mature root stock (Lynch, 1995). This implies that there must be a considerable flow of information between lateral roots and the main roots. One means by which information is transmitted from the main roots to lateral roots is via polar auxin transport along the main root, which is known to be an essential signal for lateral root initiation (Reed et al., 1998). It can be hypothesized that, lateral roots which make up the majority of the absorbing surface of roots and are therefore instrumental in the perception of environmental cues, may send signals back to the main roots and thereby influence the status of the main root. Although this flow of information from the lateral root system would be expected to influence gene expression and thus protein accumulation in the main roots, little is known about this communication.

In this study the lrtl mutant, which does not initiate lateral roots, was used as a model to study the transfer of information from lateral roots to the primary root system. The accumulation of proteins in 9-day-old primary root systems that include normal lateral roots (wild-type) was compared with the accumulation of proteins in primary root systems that do not include lateral roots (lrtl). This study demonstrates for the first time that the absence of lateral roots has a considerable impact on the composition of the proteome of the primary root. Specifically, $10 \%(15 / 150)$ of detected proteins accumulated in $l r t l$ primary roots to levels that were at least three times higher than in wild-type primary roots, providing evidence of communication from the lateral roots to the primary root system.

\section{Material and methods}

\section{Plant material}

The mutant $l r t 1$ was initially isolated from the $\mathrm{F}_{2}$-generation of an EMS-mutagenized B73 population (Hochholdinger and Feix, 1998). Heterozygous wild-type plants were selfed over three subsequent generations. Primary roots of $l r t 1 \mathrm{mu}-$ tant seedlings for the experiments described here were harvested from segregating families of the $\mathrm{F}_{5}$-generation, while the primary root system of wild-type seedlings was collected from homozygous wild-type families of the $\mathrm{F}_{5}$-generation. Each primary root protein extract was obtained from a pool of approximately 40 primary roots. Each pool consisted of 10 roots from each of four different families. Biological replicates were generated from independent root samples from different families. Proteins were isolated from complete primary roots that were cut off $1 \mathrm{~mm}$ below the coleorhiza and included the primary root tip. Wild-type and lrtl seedlings were grown in paper rolls according to Hetz et al. (1996) at $28{ }^{\circ} \mathrm{C}$ in dark.

\section{Maize primary root protein isolation}

Total proteins were isolated via acetone precipitation from primary root systems of 9-day-old wildtype and $l r t l$ seedlings according to Damerval et al. (1986) as described in Hochholdinger et al. (2004b). Dried protein pellets were resuspended in a solution containing $7 \mathrm{M}$ urea, $2 \mathrm{M}$ thiourea, $4 \%$ CHAPS, 2 mM TCIP (Pierce, Rockford, IL), 0.5\% Bio-Lytes 3/10 (Biorad, Hercules, CA), $40 \mathrm{mM}$ Tris, and $0.001 \%$ orange $\mathrm{G}$ dye.

\section{Two-dimensional separation of total primary root proteins and MALDI-TOF mass spectrometry}

Isoelectric focusing of protein extracts from maize primary roots was performed with the IPG Phor isoelectric focusing unit (Amersham Pharmacia Biotech, Uppsala, Sweden) using $18 \mathrm{~cm}$ immobilized, linear $\mathrm{pH}$ 4-7 gradients. Proteins were then separated according to their $M_{\mathrm{r}} s$ on $12-18 \%$ SDS polyacrylamide gradient gels, stained with a modified colloidal Coomassie blue stain and scanned with a Biorad GS 710 scanner (Biorad, Hercules, CA) as described previously (Hochholdinger et al., 2004b). Images were quantified and normalized with Biorad quantity one and PDQuest software prior to comparison of respective spots on wild-type and $l r t 1$ gels. Preparation of the samples for MALDI-ToF mass spectrometry was performed as previously described (Hochholdinger et al., 2004b). Crystallized proteins were analyzed with a Voyager- 
DE PRO mass spectrometer (PerSeptive Biosystems, Framingham, MA).

\section{Analysis of spectrometric data}

Proteins were identified via peptide mass fingerprints using the MS-fit program of the protein prospector package (Clauser et al., 1999; http:// prospector.ucsf.edu/) and databases NCBInr, pdbEST others, the maize EST contig database ZMtuc (www.maizegdb.org) (all databases as of 03.26.2004) and assembled genomic sequences (MAGIs) from Version 3.1 of the ISU maize genome assembly project (www.plantgenomics.iastate.edu/maize). Mass spectrometry utilities (MSU) software was used to automate the MS-fit identification tools (Porubleva et al., 2001). A protein identified via the MS-fit database search was accepted as correct only if all criteria defined by Hochholdinger et al. (2004b) were met (see footnotes of Table 1). Sequence similarity searches for EST and genomic sequences were performed using the blastx program (Altschul et al., 1997).

\section{Comparisons of different maize proteomes}

Maize protein sequences identified from the NCBInr protein database were directly compared to the maize proteins from the datasets of Chang et al. (2000) and Porubleva et al. (2001) as described in Hochholdinger et al. (2004b). The protein sequences from Chang et al. (2000) and Porubleva et al. (2001) were downloaded from Genbank using the protein accessions provided in these manuscripts. The maize protein dataset from the current study was set as the query file to search these two maize data sets using blastp with an $E$ value cut-off of $1 \mathrm{e}-10$.

\section{Results}

purification and solubilization of maize primary root proteins

Total soluble root proteins were isolated from the primary root systems of 9-day-old wild-type and lrt 1 maize seedlings. At this stage, lateral roots had already formed on the wild-type primary roots (Figure 1). Consistent with the known phenotype (Hochholdinger and Feix, 1998), seedlings homo- zygous for the lateral root initiation mutant lrt 1 did not form any lateral roots. Isoelectric focusing of total soluble primary root protein extracts was performed on a linear gradient: $\mathrm{pH}$ 4-7. After isolelectric focusing, proteins were separated according to their masses in a second dimension and stained with Coomassie blue. A total of 150 protein spots were visible including several very faint spots, which were not analyzed spectrometrically. Each map was made in triplicate from independent primary root protein preparations and excellent reproducibility was obtained (data not shown). The comparative two-dimensional electrophoresis maps of wild-type and $l r t 1$ primary root proteins are shown in Figure 2. These maps are synthetic match sets created with PDQuest software. Each map is composed of the three gels for wild-type and lrt 1 protein extracts and represents average spot intensities as calculated by PDQuest after filtering and smoothening the original scans. The intensities of all spots on a gel were normalized in silico with PDQuest to compensate for non-expression related variations in spot intensity. Average normalized spot intensities from the gels are provided in Table 1. Overall, excellent reproducibility of spot intensities was obtained among the three replicates of a genotype; the average coefficient of variance per spot for wild-type and $l r t 1$ gels was $42 \%$ and $35 \%$, respectively, which are similar to those observed by Chang et al. (2000) and in a previous dataset from our laboratory comparing maize mitochondrial N- and T-cytoplasm proteins (38\% and 39\%) (Hochholdinger et al., 2004b). As expected, less abundant proteins often had higher \% CVs.

\section{Identification and functional annotation of primary root proteins}

The 96 most abundant proteins were eluted from a representative two-dimensional gel of the mutant lrt 1, digested with trypsin and analyzed using a MALDI-ToF mass spectrometer according to the strategy described in Hochholdinger et al. (2004b). Automated MS-fit software was used for searching protein databases on all available higher plant proteins (streptophyta) since the maize genome has not yet been completely sequenced and many genes are highly conserved among higher plants.

Sixty seven of the 96 analyzed proteins were identified by matching known proteins or translated 


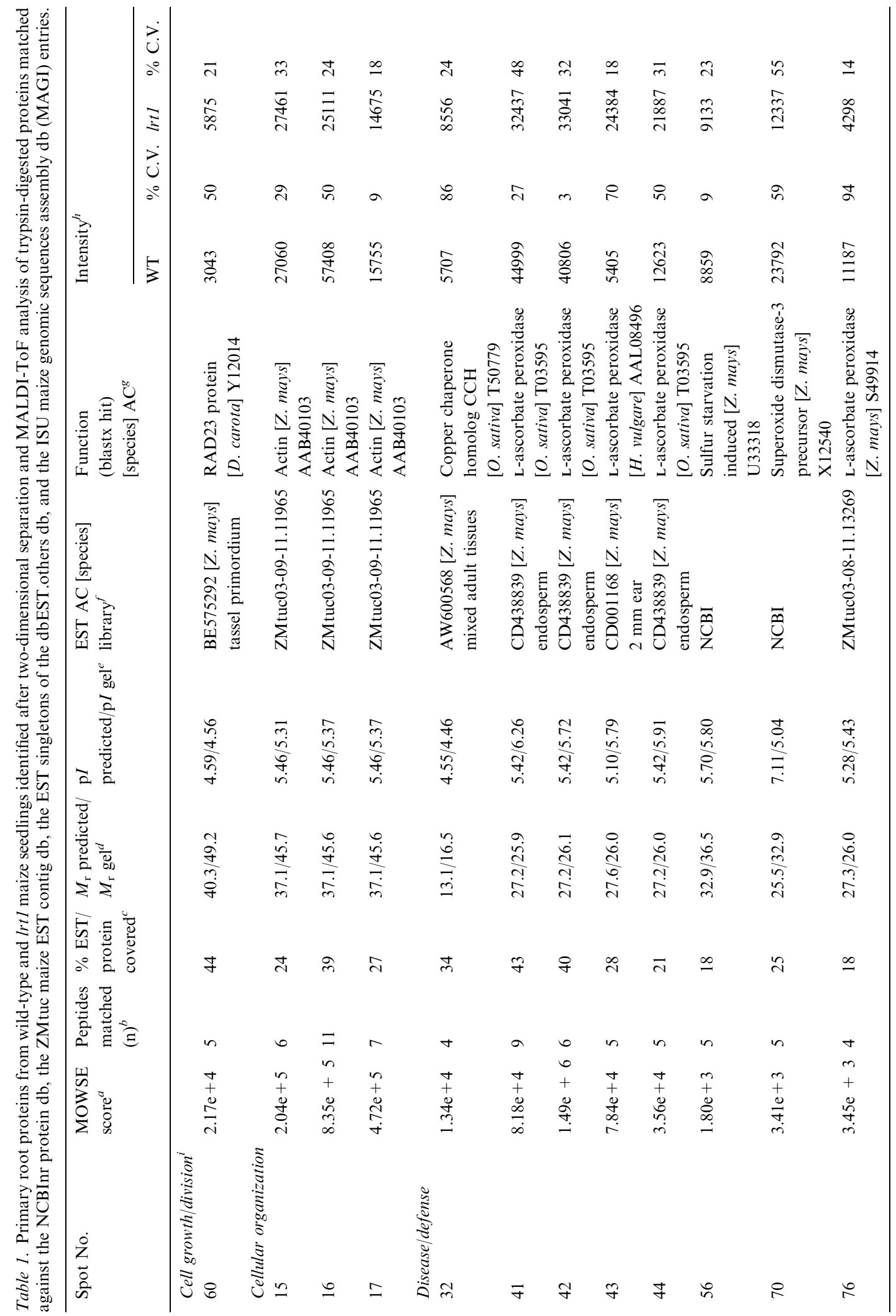




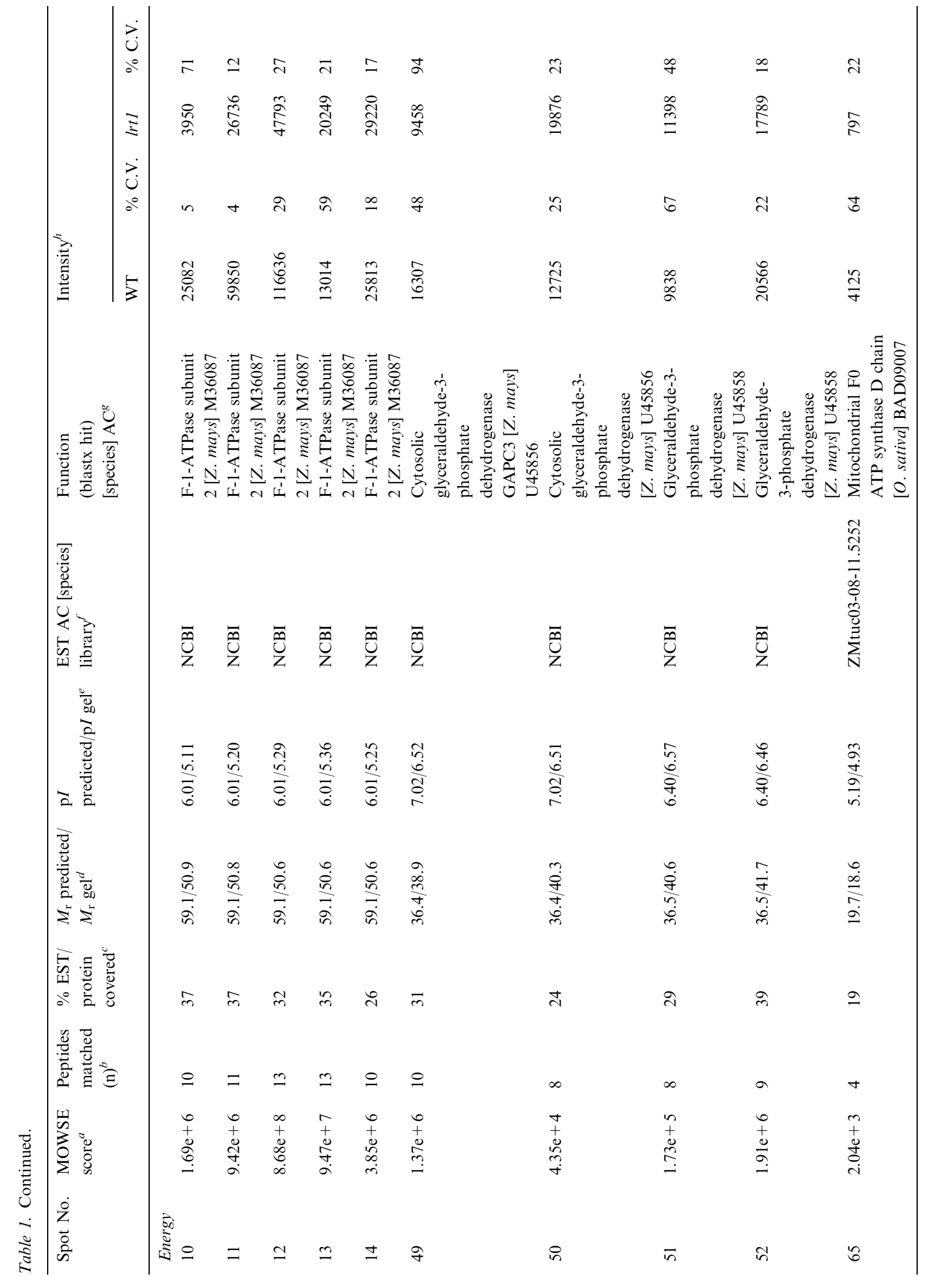




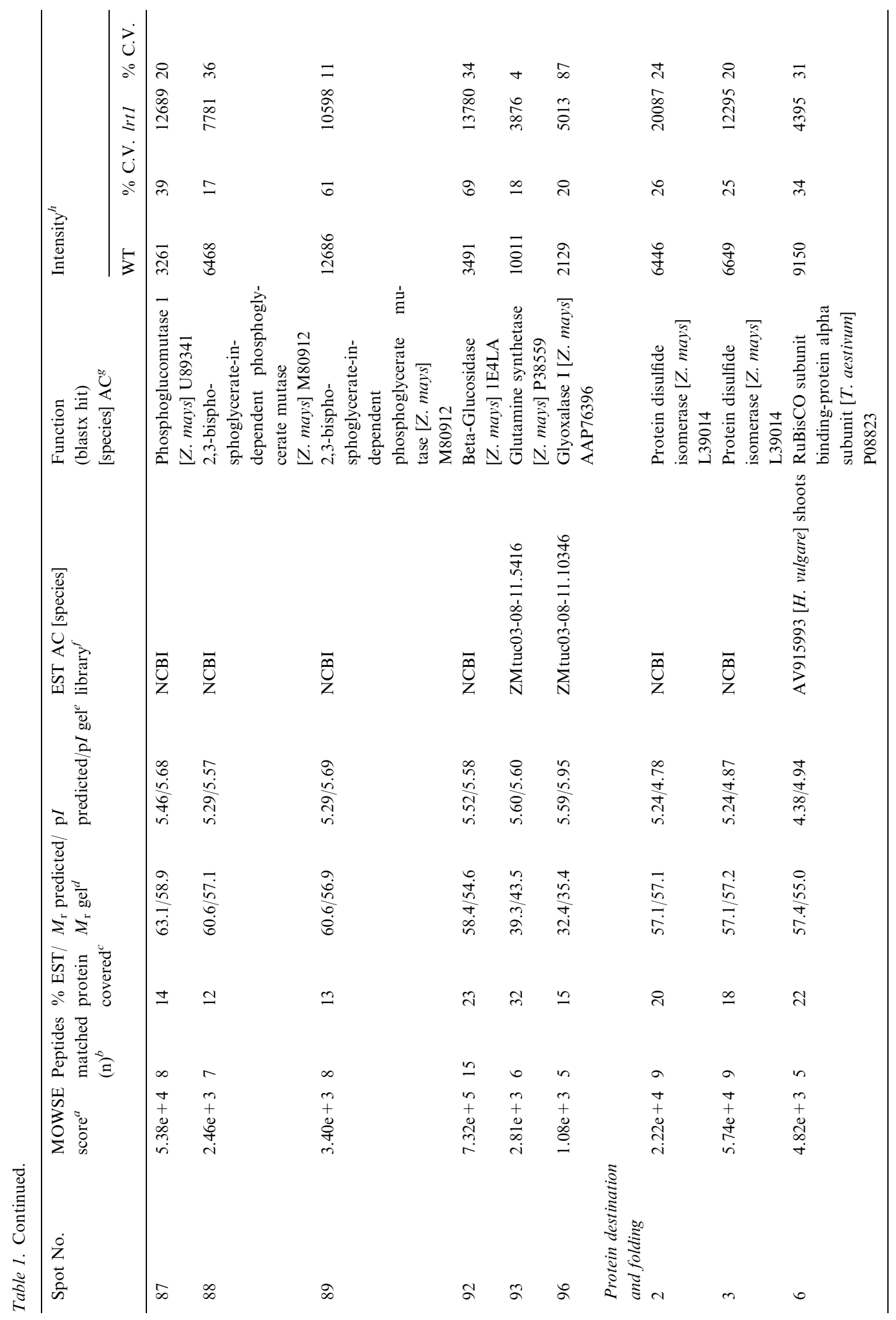




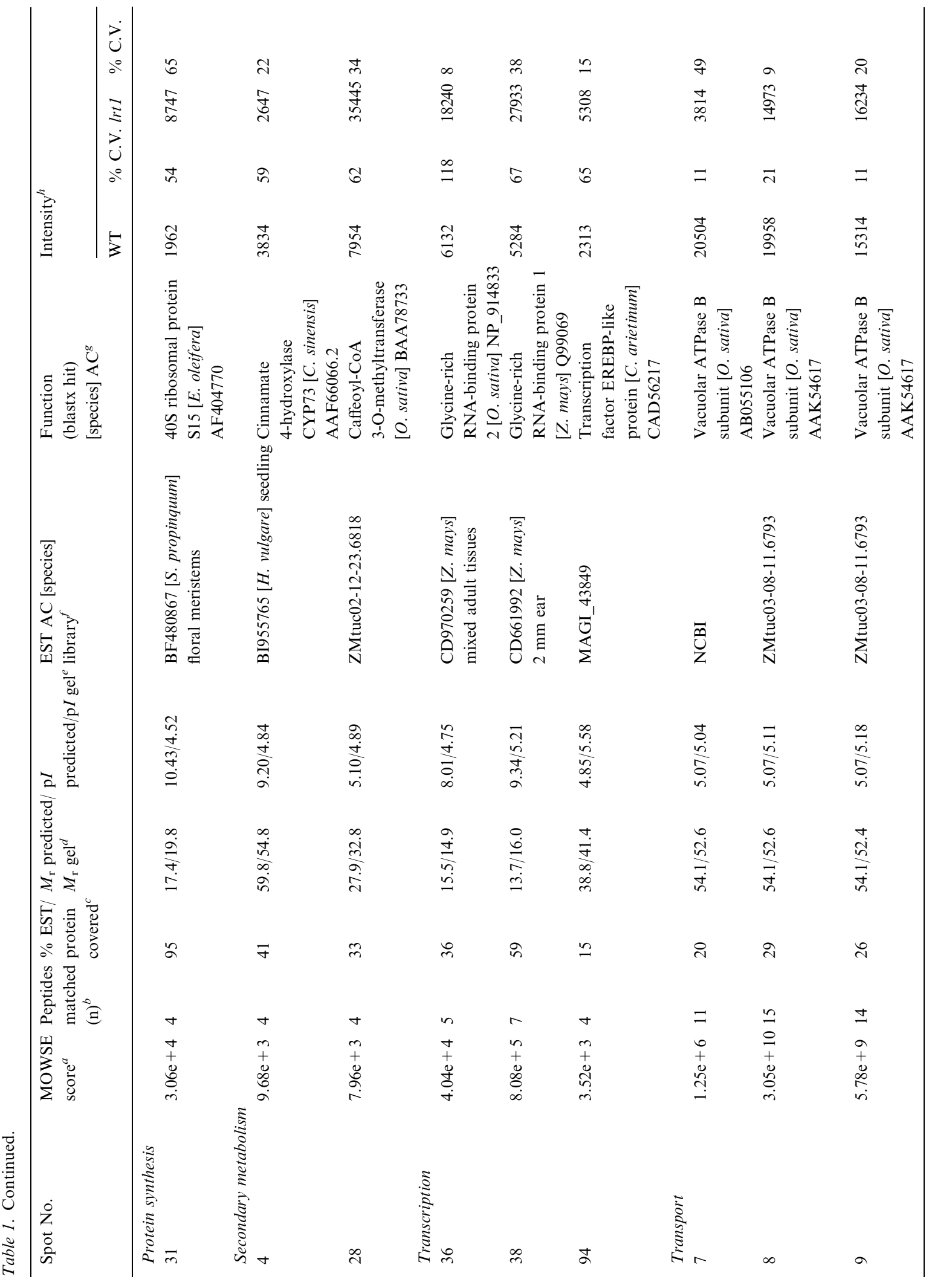




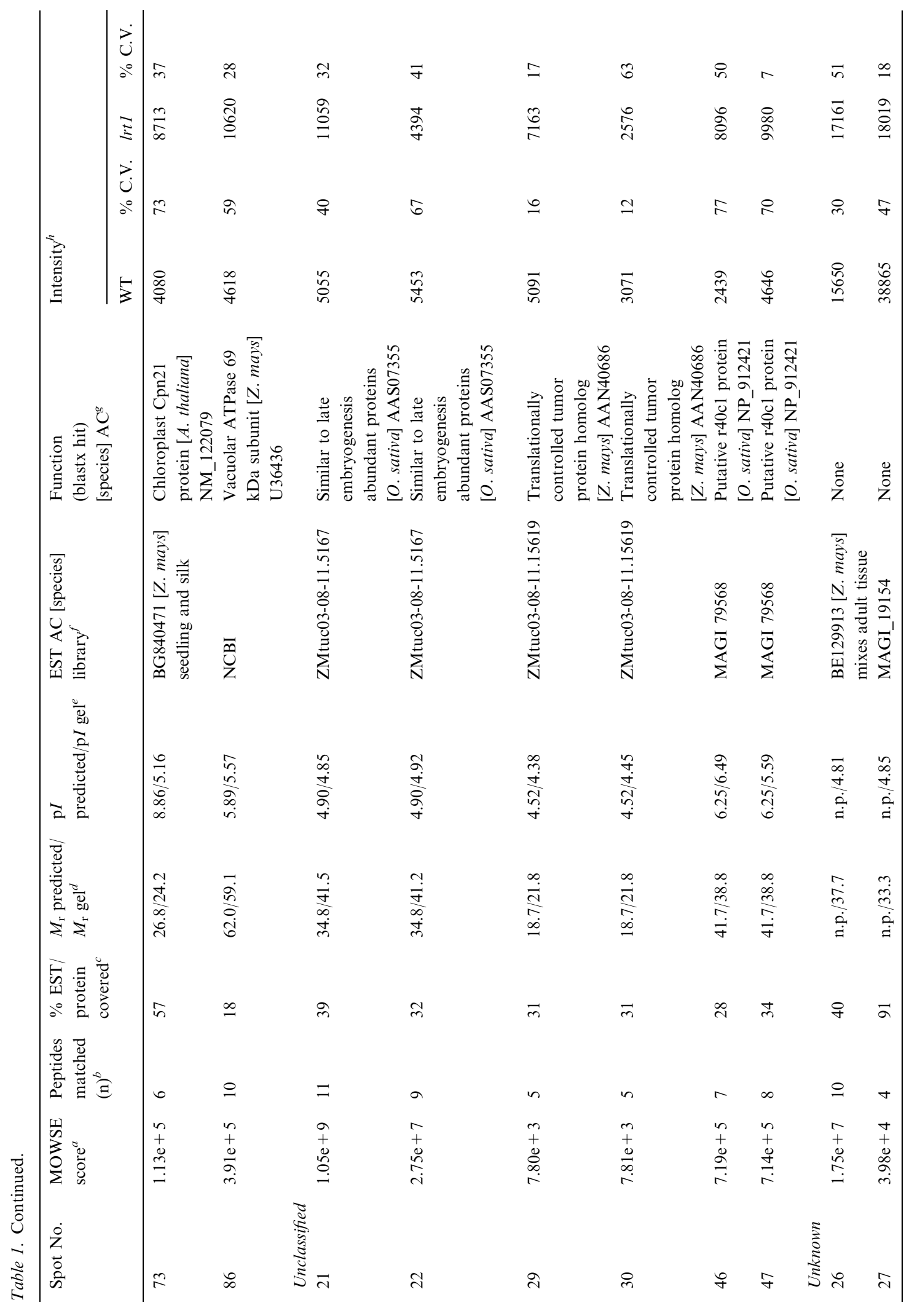




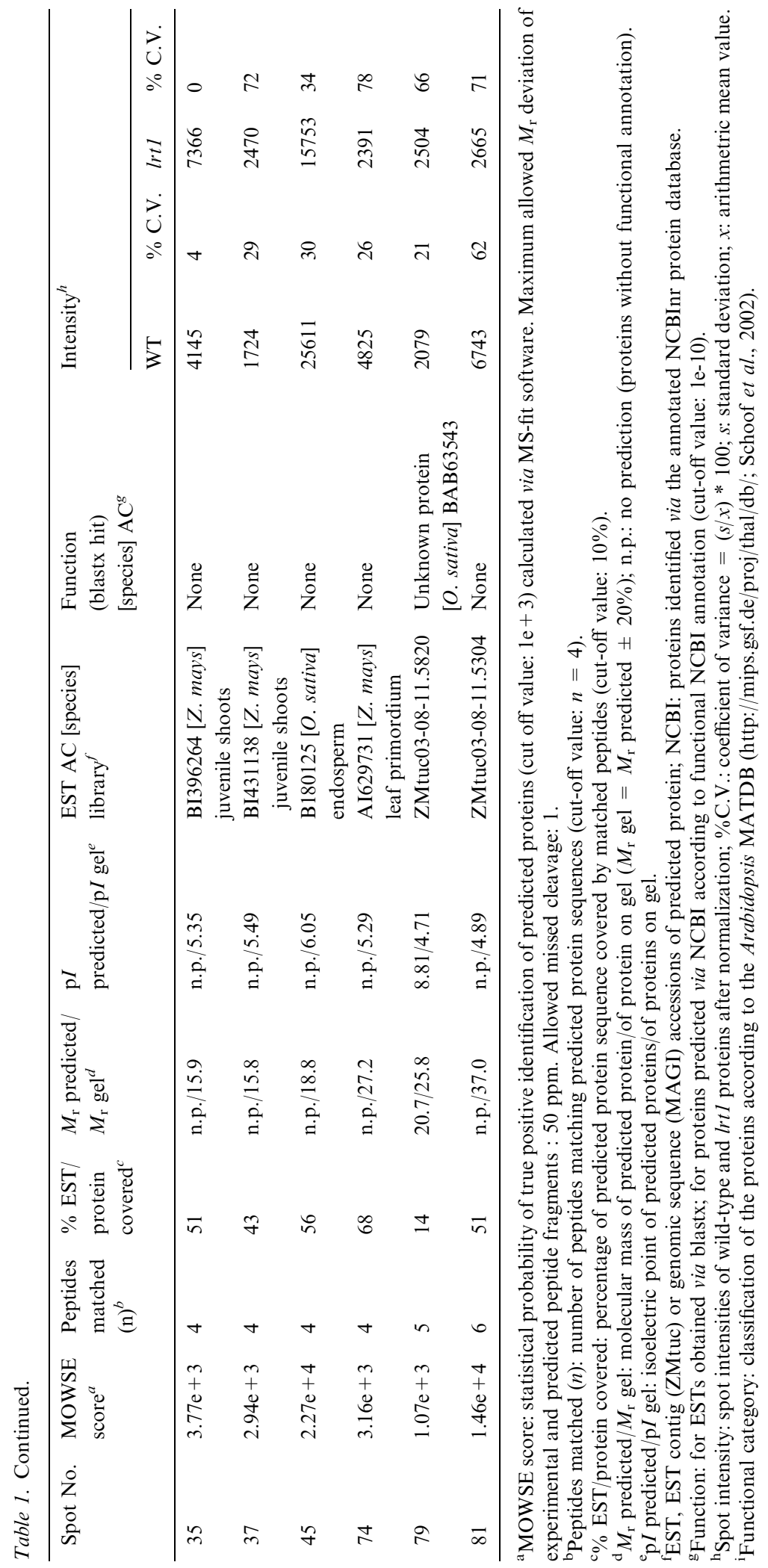



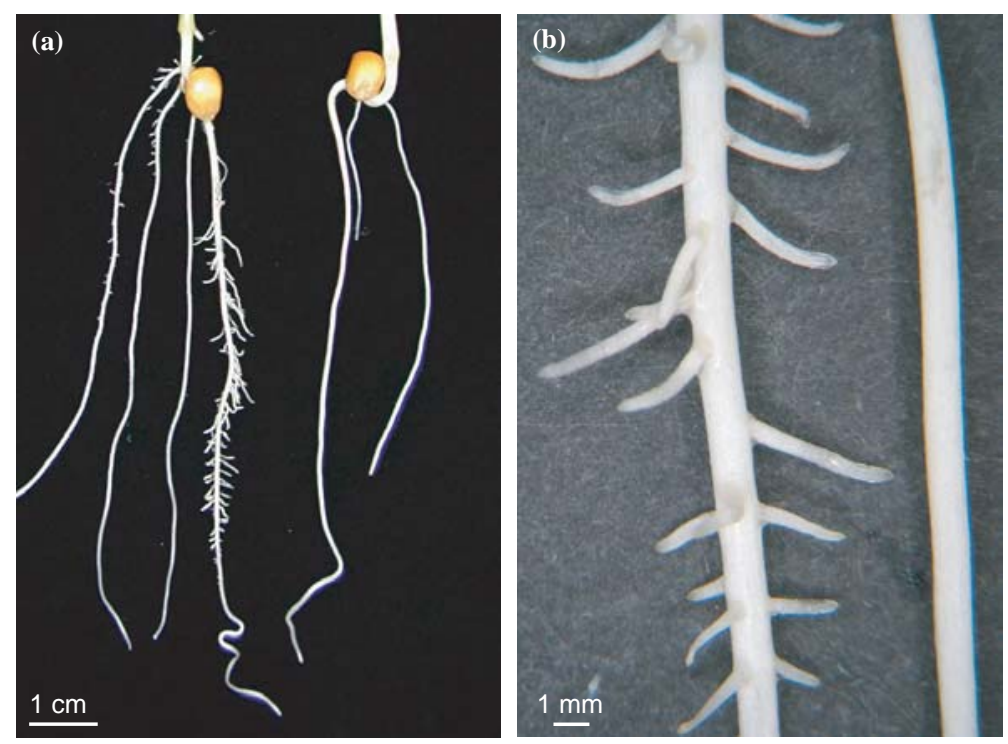

Figure 1. Root system of 9-day-old wild-type and $\operatorname{lrt} 1$ seedlings (a) left: wild-type, right: $1 r t 1$. Close up (b) showing lateral root formation in the wild-type seedling (left) and absence of lateral roots in the lrtl mutant (right).

ESTs from plants via a combination of mass spectrometry and database searches representing 47 different Genbank accessions. Table 1 summarizes the results of the MS fit database searches. The proteins are sorted according to their functions. Apparent molecular masses $\left(M_{\mathrm{r}}\right)$ and isoelectric points $(\mathrm{p} I)$ of the proteins according to the position of the proteins on the gel (Figure 2) are compared with theoretically calculated molecular masses. The function of the EST and genomic sequences was evaluated by blastx database searches. It was possible to identify 43 of the 47 accessions by known maize proteins or maize ESTs. The remaining proteins were identified via sequence similarity to proteins or ESTs from other monocotyledonous plants.

For most proteins identified via the annotated NCBInr database (Table 1) a function could be immediately predicted. No function could be proposed, however, for most proteins identified via ESTs (Table 1). These ESTs of unknown function were subjected to blastx searches to assign a function to them. Overall, functions could be assigned to 39 of the 47 identified Genbank accessions.

\section{Proteins preferentially accumulated in 1rt1 primary roots}

The primary purpose of this study was to identify proteins that preferentially accumulated in primary roots that lack lateral roots (lrt1). Proteins that exhibit such preferential accumulation in this experiment are presumably responding to direct or indirect signals from the lateral roots in wild-type plants. Protein accumulation of identified spots on normalized gels was quantified and the average intensity of these spots on three replicate gels was compared between wild-type and lrt1 primary roots.

The wild-type primary roots used in these analyses included lateral roots (i.e., lateral roots were not manually excised prior to protein extraction). The presence of lateral root proteins in the wild-type samples could potentially cause two problems. First, proteins that accumulate to very high levels in lateral roots could potentially be identified as being differentially expressed in wildtype vs. $\operatorname{lr} 1$ mutant proteomes. This was not a serious concern because our goal was instead to identify proteins that accumulated preferentially in the absence of lateral roots (i.e., that were upregulated in the lrt 1 mutant). The second potential problem with the presence of lateral root proteins in the wild-type root samples is that of 'dilution'. Specifically, if the lateral roots comprise a large proportion of the root system, lateral root proteins could reduce the relative contribution of some primary root proteins in the wild-type samples to the degree that these proteins would appear to be 

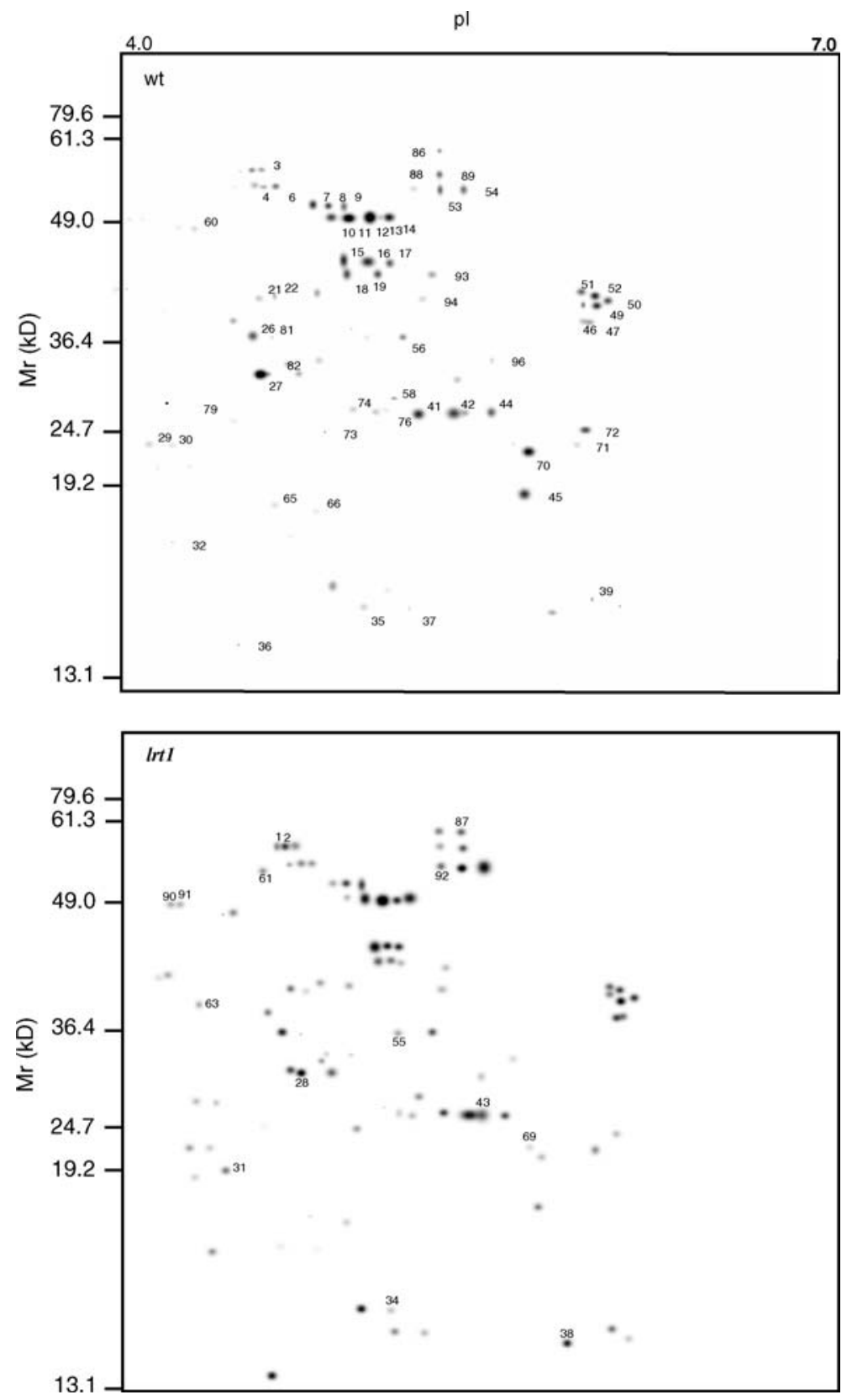

Figure 2. Comparative proteomic two-dimensional maps of CHAPS-soluble proteins extracted from 9-day-old maize primary roots of wild-type and the lateral root initiation mutant $l r t l$ seedlings showing average spot intensities. Maps for each genotype were prepared from three two-dimensional gels conducted using three independent protein preparations. Proteins were separated in the first dimension according to their $\mathrm{p}$ Is on IPG strips $\mathrm{pH}$ 4-7 and in the second dimension according to their molecular masses on linear $12-18 \%$ SDS-polyacrylamide gradient gels. Proteins were stained with colloidal Coomassie blue G250. Protein spots that were identified via MALDI-ToF spectrometry are numbered on the maps. Proteins preferentially accumulated in lrt 1 primary roots are numbered on the $l r t 1$ gel. Normalized data from these maps are summarized in Tables 1 and 2.

up-regulated in the $\operatorname{lrt} 1$ samples. To address this concern, we determined the contribution of lateral roots to the wild-type root samples. The primary and lateral roots of wild-type seedlings had average fresh weights of $104 \mathrm{mg}( \pm 19 \mathrm{mg})$ and $9 \mathrm{mg}$ ( $\pm 6 \mathrm{mg}$ ) per seedling, respectively. Hence, the 
lateral root proteins would be expected to contribute on average only to $\sim 8 \%$ of the wild-type root proteome. Hence, the 'dilution effect' described above would be expected to have only a modest impact on the relative abundance of proteins from the wild-type and $l r t l$ samples. We dealt with the potential 'dilution effect' of the wild-type root samples with lateral root proteins by considering proteins as accumulating preferentially only if their abundance levels in the lrt 1 proteome were at least three times higher than in the wild-type proteome and if they did not have overlapping standard deviations.

Even using this conservative 3-fold cut-off level, 15 of the 150 detected proteins accumulated preferentially in $l$ rt 1 primary roots (Table 2 ). Hence, $10 \%(15 / 150)$ of the detected proteins accumulated preferentially in the primary roots of $\operatorname{lrt} 1$ mutant seedlings that do not accumulate lateral roots.

Eight of these 15 proteins could be identified. They include metabolic proteins (1,4-benzochinone reductase, caffeoyl-CoA-3-O-methyltransferase, beta-glucosidase, phosphoglucomutase1) as well as proteins potentially involved in transcription (glycine-rich protein), related to plant defense (Lascorbate peroxidase), and protein synthesis and destination (40S ribosomal protein S15; protein disulfide isomerase). Four of the eight proteins that accumulate preferentially in the lrt 1 primary roots may be related to lignin metabolism (caffeoyl-CoA3-O-methyltransferase, beta-glucosidase, L-ascorbate peroxidase, 1,4-benzochinone reductase).

Three proteins (different F- and V-type ATPase subunits: spots $7,10,65)$ accumulated preferen- tially in wild-type primary roots. Because the primary roots of wild-type seedlings contained lateral roots these proteins might simply be preferentially expressed to high levels in lateral roots. For this reason and because the analysis of such proteins was outside the scope of this study, they will not be discussed further.

\section{Comparison of the maize primary root proteome with other datasets from maize}

The sequences of the maize primary root proteins presented in Table 1 were compared to the sequences of the only two other proteome data sets that are available for maize seedlings (Table 3 ). Five of 33 distinct proteins (10 protein spots) from the Chang et al. (2000) root tip data set exhibited a high degree of similarity to one or more proteins in our total primary root data set. Similarly, among 109 distinct proteins 20 (represented by 45 spots) from the Porubleva et al. (2001) green seedling leaf data set exhibited a high degree of similarity to one or more proteins in our total primary root data set. Hence, $15 \%$ of the root tip proteins identified by Chang et al. (2000) and $18 \%$ of the leaf proteins identified by Porubleva et al. (2001) were similar to proteins detected in the 9 dag maize primary root proteome. Homologs of five of 47 proteins identified in our primary root extract were found in both, the proteomes of root tips and green leaves of maize seedlings. Hence, in this maize primary root proteome dataset, $57 \%$ of the proteins (i.e., 27 proteins) encoded by distinct Genbank accessions

Table 2. Proteins that preferentially accumulated in $l r t 1$ primary roots ${ }^{\mathrm{a}}$.

\begin{tabular}{lcl}
\hline Spot No. $^{\text {b }}$ & Specificity $^{\mathrm{c}}$ & Function (AC) \\
\hline 69 & 16.4 & Putative 1,4-benzoquinone reductase BAB92583.1 \\
38 & 5.3 & Glycine-rich RNA binding protein Q99069 \\
43 & 4.5 & L-ascorbate peroxidase AAL08496 \\
31 & 4.5 & 40S ribosomal protein S15 AF404770 \\
28 & 4.5 & Caffeoyl-CoA 3-O-methyltransferase BAA78733 \\
92 & 4.0 & Beta-Glucosidase 1E4LA \\
87 & 3.9 & Phosphoglucomutase1 U89341 \\
2 & 3.1 & Protein disulfide isomerase L39014 \\
\hline
\end{tabular}

${ }^{\text {a}}$ Proteins were only considered to be differentially expressed if they accumulated to levels that were at least 3-fold different and their standard deviations did not overlap. Three proteins accumulated preferentially in the wild-type primary root systems. All of these proteins were identified as different F- and V-type ATPase subunits (spots 7, 10, 65).

${ }^{\mathrm{b}}$ The unidentified spots 1, 34, 55, 61, 63, 90 and 91 also accumulated preferentially in $\operatorname{lr} t 1$ roots.

${ }^{\mathrm{c}}$ Specificity indicates the ratio of accumulation of a particular protein between $\operatorname{lrt} l$ vs. wild-type primary root protein preparations. 
Table 3. Similar proteins identified in the proteomes of maize seedlings.

\begin{tabular}{llll}
\hline This study & Function & $\begin{array}{l}\text { Maize root tips } \\
\text { Chang } \text { et al., (2000) }\end{array}$ & $\begin{array}{l}\text { Maize leaves } \\
\text { Porubleva } \text { et al., (2001) }\end{array}$ \\
\hline $10,11,12,13,14$ & F-1-ATPase subunit 2 & $1,2,26,27,28,29,30,31,201$ \\
$15,16,17$ & Actin & 4 & $37,38,39,40,56,57,58^{\mathrm{a}}$ \\
$49,50,51,52^{\mathrm{a}}$ & Glyceroldehyde-3-phosphate dehydrogenase & $5,6,7,13^{\mathrm{a}}$ & $206,207,208,209,210,211^{\mathrm{a}}$ \\
88,89 & 2,3-bisphosphoglycerate-independent & $14,20,27,38$ & 7 \\
& phosphoglycerate & & 14 \\
6 & RuBisCO subunit binding-protein & & 23 \\
7 & alpha subunit & & 43 \\
$18,19,93^{\mathrm{a}}$ & Vacuolar ATPase B subunit & & $70,71,72,143$ \\
26,81 & Glutamine synthetase & & 160 \\
32 & Unknown & & $73,74,75,77,90,165,168,169,170$ \\
$36,38^{\mathrm{a}}$ & Copper chaperone homolog CCH & & 229 \\
39 & Glycine-rich RNA-binding protein & & 102,103 \\
$41,42,43,44,76^{\mathrm{a}}$ & Nucleoside diphosphate kinase & L-ascorbate peroxidase & 123,127 \\
73 & Chloroplast Cpn21 protein & \\
\hline
\end{tabular}

${ }^{a}$ These proteins represent more than one Genbank accession.

were not detected in either of the two other maize seedling proteomes.

\section{Discussion}

\section{Analysis of the root proteome of maize}

To date, the analysis of differential gene expression in maize roots has mainly been restricted to the analysis of a few genes identified via various methods of differential gene expression on the RNA level (e.g. Goddemeier et al., 1998; Matsuyama et al., 1999a, b; Ponce et al., 2000; Bruce et al., 2001). In this study, the soluble proteomes of 9-day-old maize primary roots harvested from wild-type and $\operatorname{lr} t 1$ seedlings which are defective in lateral root initiation were analyzed. Sixty-seven proteins, which represent 47 different Genbank accession numbers, were identified. The minimal overlap (only five shared proteins) between the major proteins identified in the primary root tip dataset of Chang et al., (2000) and our whole-root dataset might be due to the influence of the differentiation status on protein accumulation; while the root tip contains mainly meristematic tissues, the 9-day-old primary root consists predominantly of differentiated tissue. Given the relative small number of proteins identified in these studies, however, this difference might also be due to the different solubilization techniques used in the two studies. Absence of lateral roots in $1 \mathrm{rt} 1$ modulates protein
accumulation in the primary root

The architecture of the primary root of wild-type and $l r t 1$ plants differ in that the lrt 1 mutant does not initiate any lateral roots on the primary root. This morphological difference is also reflected in the protein accumulation profiles of wild-type and mutant primary roots. $10 \%$ of the proteins detected in our study $(15 / 150)$ preferentially accumulate in $l r t 1$ roots.

The increased abundance of these proteins in the primary root of a mutant that does not initiate lateral roots indicates that direct or indirect communication between the lateral roots and the primary root regulates the proteome of the primary root.

The finding that several abundant proteins are significantly up-regulated in the mutant $l r t 1$ could be explained by the following model: signals originating in the lateral roots of wild-type seedlings might either directly or indirectly repress certain genes in the primary root. According to this hypothesis, the signaling pathway between lateral and primary roots is interrupted in $l r t l$ due to this mutant's lack of lateral roots. Thus, signals that originate from lateral roots and that usually suppress the transcription of certain genes in the primary root are missing in $l r t 1$. In the absence of repression, these genes are transcribed and translated in the primary root of $l r t 1$. 
The casparian strip of the endodermis, which provides a barrier to the apoplastic transport of water and solutes (Hose et al., 2001), is composed of primary cell wall components including lignin. Plants are thought to alter the chemical compositions of their casparian strip during development or in reaction to changing environmental conditions, thereby fine-tuning its resistance to the radial flow of water and nutrients (Hose et al., 2001). Hence, given the role of the lateral roots in the uptake of water and nutrients, the casparian strip is a potential target for the signals that originate in the lateral roots and that modulate the proteome of the primary root (Hose et al., 2001). These signals could be involved in the modification of the chemical composition of the casparian strip (e.g., the lignins) to alter its capacity for the uptake of water and nutrient.

Consistent with this hypothesis, four of the eight identified proteins that were shown to accumulate preferentially in the $l r t 1$ mutant can be linked to lignin metabolism. A caffeoyl-CoA 3-O-methyltransferase accumulated to levels 4.5 times higher in the lrt 1 primary root system than in the wild-type primary root system. This enzyme plays a crucial role during the syntheseis of monolignols which later polymerize to form lignin (Boerjan et al., 2003). A beta-glucosidase accumulated 4.0 times higher in the $l r t 1$ primary roots. After synthesis of the monolignols these lignin precursors are transferred into an inactive storage or transport form designated monolignol-4- $O$-beta-D-glucosides. Beta-glucosidases regulate storage and mobilization of these monomers for lignin biosynthesis (Boerjan et al., 2003). An L-ascorbate peroxidase preferentially accumulated in lrt 1 primary roots. After transport and activation of the lignin monomers to the cell wall, lignin is formed through dehydrogenative polymerization of the monolignols (Boerjan et al., 2003). Peroxidases are one class of enzymes thought to be involved in this process (Boerjan et al., 2003). Plants contain multiple peroxidases, e.g., the Arabidopsis genome contains 73 different peroxidase genes (Boerjan et al., 2003); it is not yet clear, however, which are involved in dehydrogenative polymerization. In wood-rotting fungi, peroxidases are involved not in lignin biosynthesis, but in the degradation of lignin (Akileswaran et al., 1999). Although it is not known whether endogenous plant peroxidases are also involved in the degradation of lignin, it appears likely that perox- idases are involved in several aspects lignin metabolism in plants. Finally, a putative 1,4benzoquinone reductase was expressed 16.4 times higher in $l r t 1$ roots as compared to wild-type roots. Interestingly, this enzyme is known to play a pivotal role in lignin degradation by wood-rotting fungi via quinone intermediates (Akileswaran et al., 1999). Significantly, many of the enzymes involved in lignin biosynthesis and degradation have multiple isoforms that are differentially regulated during development (Boerjan et al., 2003).

This is the first study to demonstrate that the absence of lateral roots has an impact on the accumulation of proteins in primary roots. Further analyses of the differentially accumulated proteins identified in this study might reveal insights into the regulation of the molecular processes involved in the interactions between lateral and primary roots. Such analyses might also help to define the developmental regulation of lignin metabolism and the molecular regulation of the composition and behavior of the casparian strip.

\section{Acknowledgements}

We thank Kent Vander Velden for advice on using his 'mass spectrometry utilities' program, Ryan Brenke (ISU) and Dieter Steinmetz (University of Tuebingen) for bioinformatic support and Michael Schwall, (Aventis Crop Science), Guenter Feix, (University of Freiburg) and Joseph Dubrovsky (University of Cuernavaca) for helpful discussions. F.H.'s research at Iowa State University was supported in part by a post-doctoral fellowship from the DAAD (German Academic Exchange Service) and by the Suedwestdeutsche Saatzucht, Rastatt, Germany. Additional support was provided by Hatch Act and State of Iowa funds. Current root research in F.H.'s laboratory is supported by the German Scientific Foundation (DFG; award HO2249/4-1), the SFB 446 and the framework program 'heterosis in plants' (award HO2249/6-1).

\section{References}

Akileswaran, L., Brock, B.J., Cereghino, J.L. and Gold, M.H. 1999. 1,4-benzoquinone reductase from Phanerochaete chrysosporium: cDNA cloning and regulation of expression. Appl. Environ. Microb. 65: 415-421. 
Altschul, S.F., Madden, T.L., Schaffer, A.A., Zhang, J., Zhang, Z., Miller, W. and Lipman, D.J. 1997. Gapped BLAST and PSI-BLAST: a new generation of protein database search programs. Nucleic Acids Res. 25: 3389-3402.

Boerjan, W., Ralph, J. and Baucher, M. 2003. Lignin biosynthesis. Annu. Rev. Plant Biol. 54: 519-546.

Bruce, W., Desbons, P., Crasta, O. and Folkerts, O. 2001. Gene expression profiling of two related maize inbred lines with contrasting root-lodging traits. J. Exp Bot. 52: 459-468.

Chang, W.W., Huang, L., Shen, M., Webster, C., Burlingame, A.L. and Roberts, J.K. 2000. Patterns of protein synthesis and tolerance of anoxia in root tips of maize seedlings acclimated to a low-oxygen environment, and identification of proteins by mass spectrometry. Plant Physiol. 122: 295-318.

Clauser, K.R., Baker, P.R. and Burlingame, A.L. 1999. Role of accurate mass measurement $( \pm 10 \mathrm{ppm})$ in protein identification strategies employing MS or MS/MS and database searching. Anal. Chem. 71: 2871-2882.

Damerval, C., Vienne, D., Zivy, M. and Thiellement, H. 1986. Technical improvements in two-dimensional electrophoresis increase the level of genetic variation detected in wheat seedling proteins. Electrophoresis 7: 52-54.

Esau, K. 1965. Plant Anatomy. John Wiley and Sons, New York.

Goddemeier, M.L., Wulff, D. and Feix, G. 1998. Root specific expression of a Zea mays gene encoding a novel glycine-rich protein, ZmGrp3. Plant Mol. Biol. 36: 799-802.

Hetz, W., Hochholdinger, F., Schwall, M. and Feix, G. 1996. Isolation and characterisation of rtcs, a mutant deficient in the formation of nodal roots. Plant J. 10: 845-857.

Hochholdinger, F. and Feix, G. 1998. Early post-embryonic root formation is specifically affected in the maize mutant lrt1. Plant J. 16: 247-255.

Hochholdinger, F., Park, W.J., Sauer, M. and Woll, K. 2004a. From weeds to crops: genetic analysis of root development in cereals. Trends Plant Sci. 9: 42-48.

Hochholdinger, F., Guo, L. and Schnable, P.S. 2004b. Cytoplasmic regulation of the accumulation of nuclear-encoded proteins in the mitochondrial proteome of maize. Plant J. 37: 199-208.

Hose, E., Clarkson, D.T., Steudle, E., Schreiber, L. and Hartung, W. 2001. The exodermis: a variable apoplastic barrier. J. Exp. Bot. 52: 2245-2264.

Jung, E., Heller, M., Sanchez, J.C. and Hochstrasser, D.F. 2000. Proteomics meets cell biology: the establishment of subcellular proteomes. Electrophoresis 21: 3369-3377.
Lynch, J. 1995. Root architecture and plant productivity. Plant Physiol. 109: 7-13.

Matsuyama, T., Yasumura, N., Funakoshi, M., Yamada, Y. and Hashimoto, T. 1999a. Maize genes specifically expressed in the outermost cells of root cap. Plant Cell Physiol. 40: 469-476.

Matsuyama, T., Satoh, H., Yamada, Y. and Hashimoto, T. 1999b. A maize glycine-rich protein is synthesized in the lateral root cap and accumulates in the mucilage. Plant Physiol. 120: 665-674.

McCully, M.E. and Canny, M.J. 1988. Pathways and processes of water and nutrient movements in roots. Plant Soil 111: $159-170$.

Ponce, G., Luján, R., Campos, M.E., Reyes, A., Nieto-Sotelo, J., Feldman, L.J. and Cassab, G.I. 2000. Three maize rootspecific genes are not correctly expressed in regenerated caps in the absence of the quiescent center. Planta 211: 23-33.

Porubleva, L., Van der Velden, K., Kothari, S., Oliver, D.J. and Chitnis, P.R. 2001. The proteome of maize leaves: use of gene sequences and expressed sequence tag data for identification of proteins with peptide mass fingerprints. Electrophoresis 22: 1724-1738.

Reed, R.C., Brady, S.R. and Muday, G.K. 1998. Inhibition of auxin movement from the shoot into the root inhibits lateral root development in Arabidopsis. Plant Physiol. 118: 1369-1378.

Schoof, H., Zaccaria, P., Gundlach, H., Lemcke, K., Rudd, S., Kolesov, G., Arnold, R., Mewes, H.W. and Mayer, K.F. 2002. MIPS Arabidopsis thaliana Database (MAtDB): an integrated biological knowledge resource based on the first complete plant genome. Nucleic Acids Res. 30: 91-93.

Varney, G.T. and Canny, M.J. 1993. Rates of water uptake into the mature root system of maize plants. New Phytol. 123: 775-786.

Wang, X.L., Canny, M.J. and McCully, M.E. 1991. The water status of the roots of soil-grown maize in relation to the maturity of their xylem. Physiol. Plant 82: 157-162.

Wang, X.L., McCully, M.E. and Canny, M.J. 1994. The branch roots of Zea. IV. The maturation and openness of xylem conduits in first-order branches of soil-grown roots. New Phytol. 126: 21-29.

Wang, X.L., McCully, M.E. and Canny, M.J. 1995. Branch Roots of Zea. V. Structural features that may influence water and nutrient transport. Bot. Acta 108: 209-219. 